\title{
Estereotipia de Gênero nas Brincadeiras de Faz de Conta de Crianças Adotadas por Casais Homoparentais
}

\author{
Elder Cerqueira-Santos - Universidade Federal de Sergipe, São Cristóvão, Brasil \\ Justin Bourne - University of Toronto, Toronto, Canadá
}

\begin{abstract}
Resumo
O objetivo deste estudo foi investigar a estereotipia de gênero durante os episódios de brincadeiras de faz de conta entre crianças adotadas por casais homossexuais masculinos. A estereotipia de gênero nas brincadeiras infantis vem sendo constatada em diversos contextos, discutindo-se suas determinações biológica, individual e cultural. Este é um estudo observacional do qual participaram 13 crianças entre 3 a 7 anos, em 16 sessões, em uma sala de brinquedos em um Day Care no Canadá. Foram registrados 123 episódios de brincadeiras, sendo estes categorizados pela formação de grupos (número de participantes e gênero); tipo e tema das brincadeiras; e uso de objetos. Foram encontradas diferenças significativas para todos os critérios que caracterizam as brincadeiras como estereotipada para gênero, corroborando achados de estudos entre crianças educadas por casais heterossexuais. Meninos apresentaram episódios em grupos maiores e temas que exigiam mais uso do espaço, enquanto meninas brincaram em grupos menores e com mais uso de brinquedos.

Palavras-chave: homoparentalidade, adoção, brincadeira, gênero
\end{abstract}

\section{Gender Stereotyping in Pretend Play among Children Adopted by Same-Sex Couples}

\begin{abstract}
The aim of this study was to investigate gender stereotyping during episodes of spontaneous pretend play among children adopted by gay male couples. Gender stereotyping in children's play has been reported in different contexts, regarding discussions on their biological, cultural and individual determination. This work is a naturalistic observational study that involved 13 children aged 3-7 years during 16 observation sessions in a Day care playroom. A total of 123 episodes were analyzed, which were categorized by group's formation (number of participants and gender); type and theme of the play; and use of objects. Significant differences were found for all criteria that characterize the play as gender-typed. These findings corroborate other studies among children raised by heterosexual couples. Boys had episodes in larger groups and themes that required more use of space, while girls played in smaller groups and used more toys.
\end{abstract}

Keywords: homoparental, adoption, children's play, gender

\author{
Estereotipo de Género en Juegos de Haz de Cuenta de Niños \\ Adoptados por Parejas del Mismo Sexo
}

\begin{abstract}
Resumen
El objetivo de este estudio fue investigar el estereotipo de género durante los episodios de juegos (Haz de Cuenta) entre niños adoptados por parejas homosexuales masculinos. El estereotipo de género en los juegos infantiles viene siendo constatado en diversos contextos, discutiéndose sus determinaciones biológica, individual y cultural. Este es un estudio de observación del cual participaron 13 niños, entre 3 a 7 años de edad, en 16 sesiones en una sala de juegos en un Day Care en Canadá. Fueron registrados 123 episodios de juegos, siendo clasificados por; formación de grupos (número de participantes y género); tipo y tema de los juegos; y uso de objetos. Fueron encontradas diferencias significativas para todos los criterios que caracterizan los juegos como estereotipo para género, corroborando resultados de estudios entre niños educados por parejas heterosexuales. Los niños presentaron episodios en grupos mayores y temas que exigían más uso del espacio, mientras que las niñas jugaban en grupos más pequeños y utilizaban más los juguetes.

Palabras clave: homoparentalidad, adopción, juegos, género
\end{abstract}

As diferenças/semelhanças de gênero vêm sendo investigadas com especial atenção aos componentes culturais envolvidos em tais performances comportamentais. Parte-se da premissa de que tais diferenças/ semelhanças vão muito além da bipolaridade de sexos (macho versus fêmea) e adquire contornos ao longo da vida dos indivíduos (Butler, 1990). Para Welzer-Lang (2001), as relações sociais entre os sexos são produto da naturalização da dominação masculina e da heteronormatividade. Essa ideia de masculinidade dominante propõe a associação do sexo/gênero e da orientação sexual na construção de um modelo hegemônico de homem e um binarismo ou dicotomia de modelos: masculino/homem e feminino/mulher.

O paradigma da diferença sexual propõe também a naturalização dos papéis sociais. Joan Scott (1989) problematiza essa naturalização. Levando-se em consideração que eles compõem o que é considerado como 
gênero, Scott (1989) contribui para o entendimento de que essa categoria é socialmente construída. Para Scott (1989), o gênero é um elemento constitutivo das relações sociais baseadas nas diferenças percebidas entre os sexos e uma primeira forma de dar significado às relações de poder. Apesar de superar a naturalização do gênero, tal perspectiva reitera a lógica dicotômica do dimorfismo, que exclui as ambiguidades. Essa essência de homem/mulher é compreendida como uma norma social que rege os corpos. Tal norma é caracterizada pela linearidade do corpo (sexo), gênero e desejo. Como uma norma, esse homem/mulher deve seguir o seu destino de acordo com sua genitália e sua sexualidade voltada para a procriação da espécie. Essa linearidade do corpo, gênero e desejo será conceituada por Butler (1990) como matriz de inteligibilidade ou ordem compulsória sexo/gênero/desejo (pênis $=$ masculino $\mathrm{x}$ vagina $=$ feminino $)$.

Nesse sentido, a socialização de crianças para papéis de gênero parece ser de fundamental importância como tema de investigação na interface de diversas áreas da Psicologia (Silva, Pontes, Silva, Magalhães \& Bichara, 2006). A estereotipia de gênero em brincadeiras infantis é definida como a tendência de estruturação de jogos seguindo regras e papéis diferenciados para meninos e para meninas (Beraldo, 1993). Assim, os grupos infantis organizam suas brincadeiras espontâneas de acordo com tais regras de gênero, o que envolve a formação de grupos segregados, uso de brinquedos específicos, papéis desempenhados, etc.

Estudos clássicos em Psicologia do desenvolvimento relatam que, apesar de uma série de mudanças nos papéis de gênero em diversas culturas, a estereotipia de gênero é encontrada no desenvolvimento infantil, especialmente em determinadas fases e estilos comportamentais - crianças pré-escolares em situações naturais (Beraldo 1993; Eibl-Eibesfeldt, 1989; Harris 1999; Lordelo, 1995; Maccoby, 1988). Nesse sentido, Silva, Pontes, Silva, Magalhães e Bichara (2006) sugerem o problema: "O que estes estudos parecem indicar é que meninas e meninos nascem diferentes e tornam-se ainda mais diferentes no decorrer dos anos pré-escolares e escolares. Se a cultura adulta está mais igualitária, por que a segregação sexual continua sendo uma marca forte da infância?” (p.115).

Alguns autores, como apontam Carvalho e Pedrosa (2002), investigam tal fenômeno a partir da ideia de que as crianças transmitem regras culturais como uma microsociedade, na qual os elementos de diferenciação e papéis de gênero são mais rígidos do que no grupo cultural do mundo adulto. Dessa forma, as crianças nem sempre transmitem as regras como no mundo adulto, mas constroem scripts que são compartilhados por elas próprias, mesmo que com uma inspiração externa.

Duveen (1993) afirma que a representação social de gênero e a identidade de gênero têm um componente ontogenético que aparece logo cedo na infância. Ou seja, tal componente envolveria um aspecto desenvolvimental que é expressado em diversas culturas e persiste ao longo do tempo e gerações (Carli \& Bukatko, 2000).

Em complemento, estudos indicam que os primeiros anos escolares parecem amplificar a estereotipia de gênero em crianças a partir de uma experimentação social que o ambiente grupal proporciona (Carvalho \& Pedrosa, 2002). Nesse sentido, entende-se que as performances de gênero compartilhadas nos ambientes sociais das crianças tornam-se regulatórias de comportamentos que são apresentados pelas próprias crianças (pelo menos em sua maioria). Coloca-se assim o que é proposto por Butler (1990) como uma norma performativa que cria expectativas e reproduz a regra majoritária.

Conforme revisão realizada por Silva et al. (2006), pesquisas observacionais sobre a brincadeira entre crianças apontam uma grande influência da estereotipia de gênero em elementos do jogo lúdico, como: preferência por temáticas, atitudes, comportamentos, percepções, escolha de parceiros, tamanho de grupos e uso de objetos/brinquedos. De modo geral, constata-se que meninos brincam em grupos maiores, em episódios mais curtos, com menos objetos e temáticas mais ativas (motoras e em ambientes mais amplos); enquanto meninas formam grupos menores, de episódios mais longos e com temáticas mais "intimistas" em ambientes menores (Carli \& Bukatko, 2000).

Nesse ponto, coloca-se a questão da influência direta dos ambientes socializadores na estereotipia de gênero em crianças pré-escolares durante suas brincadeiras de faz de conta. Um grupo específico e de bastante singularidade na sua forma de socialização é o de crianças adotadas por casais homoafetivos (homoparentais). Como tais crianças mantêm e/ou flexibilizam os papéis de gênero a partir dos diversos modelos parentais?

Absolutamente, tal pergunta não pode ser seguida de um julgamento sobre as possíveis diferenças/similaridades encontradas, mas abre discussão sobre a estereotipia de gênero em crianças frente às mudanças significativas nos modelos adultos e na bipolaridade e 
conformidade sexo/gênero. Diversas configurações familiares existem há bastante tempo, como crianças educadas por avós, mães solteiras, dentre outras, no entanto, o fenômeno da adoção homoparental vem levantando discussões em diversos países, levando à criação de novas leis e controvérsias no debate leigo. Nesse sentido, é fundamental que estudos acompanhem tais configurações familiares e produzam um corpo de conhecimentos que sustentem argumentos sólidos embasados em achados empíricos. O objetivo deste estudo foi investigar a estereotipia de gênero nas brincadeiras de faz de conta de crianças, filhos (as) adotivos (as) de casais homoparentais em uma situação de day care (creche/berçário). Parte-se de um pressuposto teórico claro de que a estereotipia é muito mais ampla do que a imitação de modelos diretos. Ou seja, as crianças sofrem múltiplas influências em seu desenvolvimento de gênero.

\section{Adoção Homoparental e Desenvolvimento Infantil}

Vários estudos recentes têm investigado a parentalidade entre casais homoafetivos, chamada homoparentalidade (Bergman, Rubio, Green \& Padròn, 2010; Murphy, 2013; Riggs \& Due, 2010), revelando um aumento desse fenômeno na comunidade de gays e lésbicas e algumas das suas repercussões. Especificamente entre homens gays, estudos revelam uma representação dominante de papéis excludentes quando se fala em paternidade e homossexualidade masculina, principalmente pela impossibilidade do casal ter um filho biológico conjunto (Berkowitz, 2009). A adoção tem sido a forma mais comum de concretizar o desejo de parentalidade entre homens gays (Stacey, 2006).

Em estudo de revisão sistemática da literatura, Cecílio, Scorsolini-Comin e Santos (2013) apontam que, no Brasil, em uma década (2000-2010), apenas dez estudos sobre a adoção homoparental foram realizados e publicados nas principais bases de dados científicas do país, sendo três artigos empíricos e sete teóricos. Os autores destacam especialmente três tendências nos estudos sobre a temática: a preocupação com as consequências da adoção para as crianças (aspectos desenvolvimentais negativos e positivos); as alternativas na busca da parentalidade; e as questões ligadas à adoção em si (legislação, mudanças históricas, etc.).

Em estudo exploratório sobre representações da adoção homoparental, Araújo, Cruz, Souza e Castanha (2007) identificaram que estudantes universitários de Direito e de Psicologia são contrários à adoção por casais homoafetivos. Segundo tal estudo, os universitários alegam justamente a melhor decisão para as crianças, ancorados em noções negativas sobre como um casal homossexual poderia educar seus filhos. $\mathrm{O}$ argumento principal seria a carência de referencial de ambos os sexos para o desenvolvimento saudável da criança.

Apesar de avanços legais (casamento homoafetivo e possibilidade legal de adoção pelo casal do mesmo sexo), poucos estudos internacionais e nenhum estudo brasileiro foram localizados que tratam do acompanhamento de crianças adotadas por casais homoafetivos com o objetivo de investigar aspectos desenvolvimentais nessas novas configurações familiares. Os poucos achados até o momento revelam não haver diferenças significativas em ajustamento psicológico ou comportamento sexual e de gênero quando comparadas crianças em famílias heteroafetivas com as em homoafetivas (Goldberg, 2010; Patterson, 2009). Embora, crianças filhas de casais homoafetivos possam passar por mais situações de dificuldade em certas interações sociais, como preconceito na escola, instituições e família extensa (Golombok et al., 2014). No entanto, existe também a hipótese de que crianças educadas sem a figura feminina apresentem comportamentos de gênero menos estereotipados (Goldberg, Kashy, \& Smith, 2012).

\section{Método}

O estudo seguiu o modelo da microetnografia de Grenn e Wallat (1981), descrito no Brasil por Sager e Sperb (1998). Utiliza-se da técnica da observação naturalística com foco em padrões de participação em episódios de brincadeira num contexto específico (descrito nos procedimentos).

\section{Participantes}

As observações foram realizadas em um contexto de Day care envolvendo 13 crianças de ambos os sexos (8 meninos e 5 meninas) com idades entre 3 e 7 anos. O Day care escolhido para esse estudo tratava-se de uma associação de casais homoparentais (apenas homens gays) no Canadá. As crianças passavam somente um turno no serviço enquanto os pais estavam em uma reunião semanal da associação, que funcionava como um grupo de apoio anônimo. Foram realizadas 16 sessões de observação, cada uma delas de 60 minutos. No total, foram contabilizados 123 episódios de brincadeira sem a participação de adultos cuidadores (as). 


\section{Situação e Procedimentos}

O sistema de Day care escolhido tratava-se de um serviço semanal vinculado a uma reunião de pais. $\mathrm{O}$ espaço era constituído de uma sala equipada com brinquedos, colchonetes, vídeo e material educativo (livros e papelaria). Havia quatro monitores que cuidavam das crianças e sugeriam atividades. Para esse estudo foram observadas brincadeiras espontâneas das crianças com ou sem uso do material disponível.

Para a coleta de dados foi realizado registro cursivo e de episódios seguindo uma ficha a ser preenchida. Dois pesquisadores realizaram os registros (um para cada técnica). A ficha de observação está descrita na seção instrumento.

Foi adotada a técnica do sujeito/grupo focal por amostragem de episódio. Um dos pesquisadores numerava mentalmente todas as crianças da sala e o outro pesquisador escolhia um número. A criança selecionada pelo número escolhido era o foco da observação, tendo seu primeiro episódio após o sorteio descrito (o que envolveu a participação de outras crianças na maioria dos casos, sendo o grupo inteiro observado). Após o final do primeiro episódio um novo número era escolhido e uma nova criança passava a ser o foco (de outro ou mesmo grupo). O tempo de cada episódio variou entre 2 e 15 minutos.

\section{Instrumentos}

Dois instrumentos foram utilizados. Uma folha de registro para anotações cursivas, que era utilizada por um dos pesquisadores, e um protocolo de registro de episódio baseado no estudo de Wanderlind, Martins, Hansen, Macarini e Vieira (2006), utilizado pelo segundo pesquisador. Tal protocolo seguia as seguintes categorias:

Tipo de interação: número de crianças no episódio: a) sozinha; b) díade ou, c) grupo.

Gênero: a) homogêneo - criança-foco brinca com parceiros do mesmo sexo; b) Grupo misto - criança-foco brinca com parceiros de sexo oposto ou de ambos os sexos.

Uso de objetos: a) uso de brinquedo - brinquedo industrializado; b) uso de objetos - sucatas, caixas, madeira ou qualquer objeto cuja função principal não seria a brincadeira; c) sem uso de brinquedos ou objetos.

Tipo de brincadeira (baseado em Morais \& Otta, 2003): a) fantasiosa - brincadeiras que incluem tratar os objetos como se fossem outros, atribuir a si e aos outros papéis diferentes dos habituais, criar cenas imaginárias e representá-las, como heróis, monstros, etc.; b) realística - situações em que a criança utiliza o faz de conta replicando e transformando uma situação da vida cotidiana, como esportes, profissões, casinha; c) turbulenta - ocorre quando a criança exibe movimentos bruscos, semelhantes aos de uma luta, porém manifestando expressão facial hilariante, ao mesmo tempo em que o oponente "atacado" não demonstra nenhuma expressão de ressentimento (atividades que envolvem luta, perseguição e fuga, provocação e zombaria, rolar em dupla no chão, encostar no colega de forma brusca); e d) jogos - inclui-se aí os jogos sociais de regras (envolvem a ritualização de papéis, um ciclo repetitivo de ações e, em geral, são competitivos).

Temas das brincadeiras: foram classificados os temas mais recorrentes a posteriori, sendo estes: casinha, profissões, transportes, super-heróis, animais, monstros e esportes.

\section{Análise de Dados}

Os dados dos protocolos foram checados a partir da leitura do registro cursivo de cada episódio a fim de verificar a precisão do registro. Em seguida, foram inseridos no software_SPPS 17 for Windows seguindo as categorias a priori descritas. Foram realizadas análises univariadas de todas as variáveis, assim como análises bivariadas (teste $t$ de Student e Qui-Quadrado) entre as características dos episódios e os grupos por sexo.

\section{Resultados e Discussão}

Dos 123 episódios observados, houve uma distribuição aleatória (pelo critério do sorteio descrito no método) de episódios registrados com foco em meninos (78 episódios) e em meninas (45 episódios). No entanto, houve diferença significativa no tempo médio de duração dos episódios envolvendo meninos $(M=$ $5,34 ; D P=3,45)$ e meninas $(M=7,89 ; D P=6,98)$ sendo $t=1,68(p=0,04)$. A diferença da média de duração é um primeiro indicador de diferenciação dos episódios por gênero, conforme apontado em diversos estudos (Beraldo, 1993). As idades das crianças-foco variaram entre 3 e 7 anos, no entanto, não houve uma distribuição igualitária por faixa etária para análise de número de episódios por idade. A amostra contou apenas com uma criança de 3 anos (menino) e a maioria entre 6 e 7 anos (9 das 13 observadas).

A Tabela 1 apresenta as diferenças nos percentuais de tipos de interação e formação de grupos por gênero para meninos e meninas. A interação apresentou 
diferença significativa, com padrão de maior percentual para brincadeiras solitárias e grupos menores entre as meninas; enquanto os meninos apresentaram maior percentual na formação de grupos envolvendo três ou mais crianças. Tais resultados estão de acordo com a maior parte dos achados sobre a formação de grupos, corroborando com a ideia de que meninas envolvem-se em episódios com grupos menores (Silva et al., 2006). Quanto à formação por gênero, não foi encontrado resultado significativo quando comparados meninos e meninas; no entanto, os percentuais para grupos homogêneos são maiores nos dois grupos, indicando uma tendência de segregação dos sexos nos grupos de episódios. Do ponto de vista desenvolvimental, tal aspecto é esperado, especialmente em pré-escolares (Silva et al., 2006). Ainda sobre a formação de grupos, observou-se que as crianças menores estavam em grupos liderados por crianças mais velhas, em geral por meninas e em episódios temáticos que contribuíam para comportamentos de cuidado, indicando certa tendência de segregação de gênero como apontada por Beraldo (1993), Carvalho e Pedrosa (2002), Sager e Sperb (1998) e Silva et al. (2006).

A Tabela 2 apresenta as diferenças percentuais para uso de objetos e tipos de brincadeiras entre meninos e meninas. Houve diferença significativa no uso de objetos, com percentual maior de uso de brinquedos entre as meninas e maior uso de objetos e episódios sem objetos entre os meninos. $\mathrm{O}$ uso de objetos pode estar relacionado ao tipo e temas de brincadeiras em cada episódio. Observou-se um percentual maior de brincadeiras turbulentas e de jogos entre os meninos, o que poderia acontecer sem brinquedos ou com objetos. Por outro lado, o maior percentual de brincadeiras fantasiosas entre as meninas pode estar relacionado ao uso de brinquedos, que serviriam como pivô do faz de conta fantasioso. A diferença para o tipo de brincadeira foi significativa entre os grupos com foco em meninos e meninas.

De acordo com Silva et al. (2006), as atividades de crianças podem estar relacionadas às normatizações e modelos adultos para os quais homens se envolvem mais em atividades realistas e mulheres em atividades abstratas. Os mesmos autores refletem que tais padrões de gênero já não são hegemônicos e mudanças culturais recentes poderiam influenciar os comportamentos infantis. Os resultados descritos aqui apresentam características normativas mesmo em uma situação social particular em termos de papéis de gênero (dois pais), uma vez que se espera que famílias homoparentais tenham mais probabilidade de flexibilização de papéis de gênero tradicionais, com divisão de tarefas domésticas e cuidados com as crianças.

Com o objetivo de investigar a diferença de temáticas das brincadeiras de faz de conta comparando-se os grupos exclusivos por sexo (aqui chamados de heterogêneo) e grupos mistos, a Figura 1 apresenta os percentuais para tipos e temas dos episódios observados. Houve diferença significativa para os tipos de brincadeiras observadas por formação de grupos por sexo $\left(X^{2}=8,66 ; p<0,0001\right)$. Os grupos homogêneos (somente meninos ou somente meninas) se engajaram mais em brincadeiras de faz de conta fantasiosos e realísticos (como heróis e monstros), enquanto os grupos mistos brincaram mais de faz de conta envolvendo jogos. Conforme esperado, as brincadeiras turbulentas (brincar de brigar) foram predominantemente em grupos homogêneos de meninos.

A segregação encontrada na formação de grupos também está de acordo com o encontrado em outros contextos (Duveen, 1993; Carli \& Bukatko, 2000), apesar de Silva et al. (2006) apontarem para uma maior

Tabela 1

Teste de Qui-Quadrado para Tipos de Interação Grupal e Segregação de Gênero entre Meninos e Meninas

\begin{tabular}{lccc}
\hline & Meninos & Meninas & $\mathrm{X}^{2}(p)$ \\
\hline Interação & $N(\%)$ & $N(\%)$ & \\
Brincadeira solitária & $14(17,94)$ & $10(22,22)$ & $8,09(0,017)$ \\
Díade & $25(32,05)$ & $24(53,33)$ & \\
Grupo (três ou mais) & $39(50,00)$ & $11(24,45)$ & \\
Formação por gênero & $N(\%)$ & $N(\%)$ & $0,18(0,660)$ \\
Homogêneo & $43(67,19)$ & $22(62,85)$ & \\
Misto & $21(32,81)$ & $13(37,15)$ & \\
\hline
\end{tabular}

Psico-USF, Bragança Paulista, v. 21, n. 1, p. 125-133, jan./abr. 2016 
Tabela 2

Teste de Qui-Quadrado para Uso de Objetos e Tipo de Brincadeira entre Meninos e Meninas

\begin{tabular}{lccc}
\hline & Meninos & Meninas & $\mathrm{X}^{2}(p)$ \\
\hline Uso de objeto & $N(\%)$ & $N(\%)$ & \\
Brinquedo & $35(44,87)$ & $31(68,89)$ & $6,77(0,033)$ \\
Objeto & $28(35,90)$ & $10(22,22)$ & \\
Sem objeto & $15(19,23)$ & $04(8,89)$ & \\
Tipo de brincadeira & $N(\%)$ & $N(\%)$ & \\
Fantasia & $23(29,49)$ & $25(55,55)$ & \\
Realística & $30(38,46)$ & $14(31,11)$ & \\
Turbulenta & $08(10,25)$ & $02(4,45)$ & \\
Jogos & $17(21,80)$ & $04(8,89)$ & \\
\hline
\end{tabular}

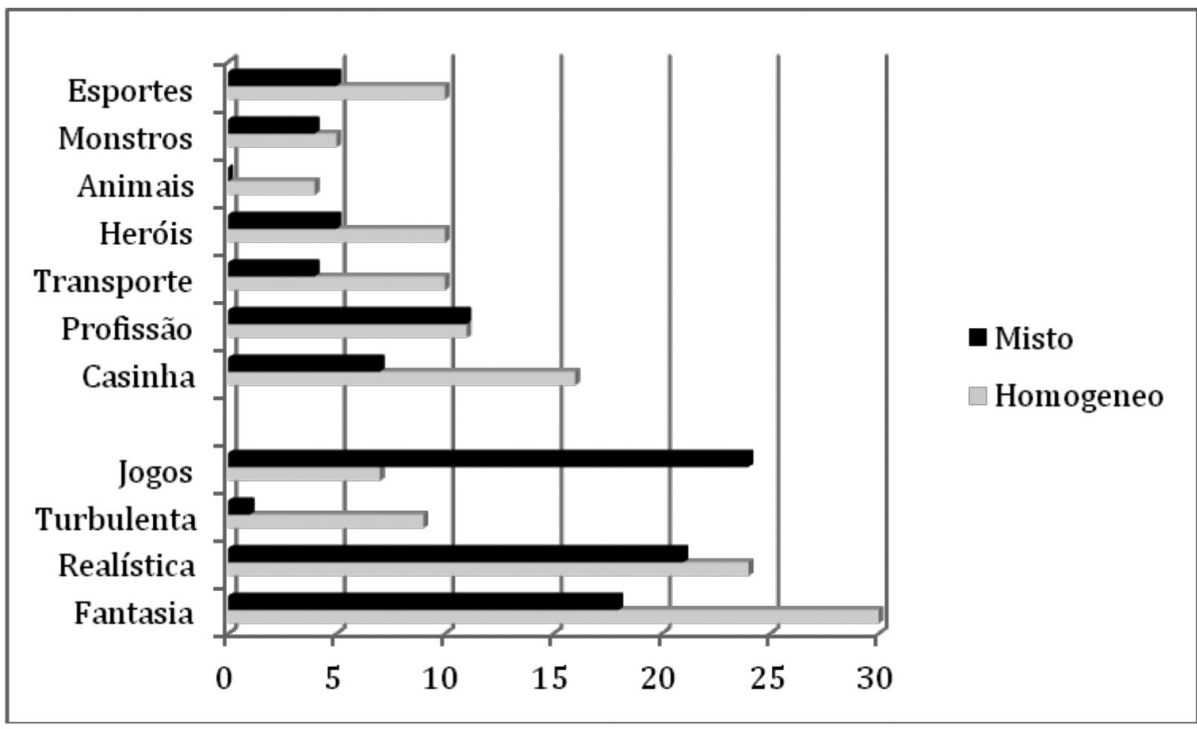

Figura 1. Frequência de temas e tipos de brincadeiras de acordo com a formação de grupos por gênero.

flexibilização. Entende-se que tal segregação pode ser aumentada pela idade (Maccoby, 1988), uma vez que a maior parte das crianças observadas tinha 6 e 7 anos. Destaca-se que os grupos heterogêneos seguiram padrões esperados em termos de combinações de idades e de tipos de brincadeiras, com crianças mais novas em episódios de cuidado por parte das mais velhas, especialmente meninas.

\section{Considerações}

Este estudo teve como objetivo investigar a estereotipia de gênero durante episódios de brincadeiras de faz de conta entre crianças adotadas por casais homossexuais masculinos. Optou-se por um método naturalístico de observação de comportamento sem a interferência de adultos. De modo geral, o estudo constatou padrões de estereotipia de gênero semelhantes aos encontrados em outros estudos com crianças educadas em ambientes familiares heterossexuais (Golombok, 2013; Patterson, 2009, Silva et al., 2006).

Tal padrão encontrado abre a discussão para o fato de que a estereotipia de gênero pouco depende do modelo parental (Carli \& Bukatko, 2000; Duven, 1993) e pode estar relacionada à interação grupal e a aspectos da macrocultura. Conforme destacado por Lordelo 
(1995), também não se pode deixar de considerar aspectos biológicos no surgimento de comportamentos diferenciados por sexo. Silva et al. (2006) ressaltam que "a base das diferenciações de gênero é biológica, mas as construções que se processam e a forma como se processam são simbólicas, são sociais" (p.114).

O debate em torno de estudos com essa temática está apenas começando, mas os achados têm apontado pouca diferença em ajustamento psicológico e padrões comportamentais entre crianças educadas por casais heteroafetivos e homoafetivos. Enquanto, outros estudos apontam até para diferenças com resultados mais positivos para as famílias homoparentais, como no estudo de Golombok et al. (2014), que aponta maior bem-estar psicológico nesse grupo de crianças.

É importante destacar que os estudos realizados até o momento com familias homoparentais, inclusive este, não buscam um julgamento dos seus achados sobre semelhanças e diferenças. Dessa forma, é essencial enfatizar que os resultados aqui apresentados (de semelhanças em padrões) não podem ser interpretados como positivos ou negativos. Mesmo que fossem encontradas mudanças na estereotipia de gênero, tais mudanças devem ser encaradas como novas formas como as crianças podem refletir mudanças sociais. Em estudos comparativos em diferentes ambientes sociais, nos quais os papéis de gênero nos adultos diferem significativamente, já foram encontradas diferenças nas brincadeiras das crianças (inclusive filhos de heterossexuais) que "replicavam" tais mudanças (Goldberg et al., 2012).

Os resultados desse estudo são limitados por uma série de aspectos. Não foi verificado, por exemplo, o ambiente da macrocultura onde essas crianças estavam inseridas, incluindo fatores da interação doméstica entre os pais (papéis de gênero dentro de casa) e elementos da família extensa (avós, tios, amigos). Apesar de ter sido realizado no Canadá, país onde as barreiras de gênero parecem mais flexíveis, o padrão heteronormativo é dominante, o que pode influenciar as próprias práticas parentais de casais homoafetivos (que podem entrar em tentativa de conformidade com a regra geral heteroafetiva).

Outra limitação importante é intrínseca aos vários estudos com crianças adotadas em famílias homoparentais. Em geral, casais gays passam por processo diferenciado de adoção, muitas vezes tornando o processo mais criterioso e mais lento. Como apontam Golombok et al. (2014), casais homoafetivos adotam crianças mais velhas e estão todo o tempo sob a observação avaliativa externa sobre o ajustamento familiar e condições da criança, podendo apresentar práticas parentais conservadoras.

Finalmente, estudos com essa temática são fundamentais para o debate recorrente sobre as consequências da adoção homoparental, colaborando para desmistificar crenças repassadas pelo senso comum sem a menor evidência empírica. Também colabora para que paradigmas psicológicos ultrapassados sejam repensados, reforçando a necessidade de debates que consideram aspectos culturais, individuais e biológicos no desenvolvimento sexual de crianças. Em um contexto de mudanças sociais que envolvem regras de gênero, como a emancipação feminina e as diversas configurações familiares, estudos empíricos sobre a homoparentalidade vêm demonstrando achados positivos que reforçam a ideia não discriminatória para a constituição de diversos desenhos de famílias.

\section{Referências}

Araújo, L. F. de, O., Cruz, J. S., Sousa, V. C., \& Castanha, A. R. (2007). Adoção de crianças por casais homoafetivos: Um estudo comparativo entre universitários de direito e de psicologia. Psicologia \& Sociedade, 19(2), 95-102. doi: 10.1590/ S0102-71822007000200013.

Bergman, K. Rubio, R. J., Green, R. J., \& Padròn, E. (2010). Gay man who become fathers via surrogacy: The transition to parenthood. Jour nal of GLBT Family Studies, 6, 111-141. doi: 10.1080/15504281003704942

Beraldo, K. E. (1993). O gênero de brincadeiras de crianças de 5 a 10 anos (Dissertação de mestrado não publicada). Instituto de Psicologia, Universidade de São Paulo. São Paulo, SP.

Berkowitz, D. (2009). Theorizing lesbian and gay parenting: Past, present and future scholarship. Journal of Family Theory and Review, 1(3), 117-132. doi: 10.1111/j.1756-2589.2009.00017.x

Butler, J. (1990). Gender Trouble. New York: Routledge.

Carli, L. L., \& Bukatko, D. (2000). Gender, communication, and social influence. A developmental perspective. Em T. Eckes \& H. M. Trautner (Eds.). The Developmental Social Psychology of Gender (pp. 295331). Mahwah, NJ: Lawrence Erlbaum Associates.

Carvalho, A. M. A., \& Pedrosa, M. I. (2002). Cultura no grupo de brinquedo. Estudos de Psicologia (Natal), 7(1), 181-188. doi: 10.1590/ S1413-294X2002000100019 
Cecílio, M. S., Scorsolini-Comin, F., \& Santos, M. A. (2013). Produção científica sobre adoção por casais homossexuais no contexto brasileiro. Estudos de Psicologia (Natal), 18(3), 507-516. Recuperado de http://www.redalyc.org/articulo. oa?id=26128793011

Duveen, G. (1993). The development of social representation of gender. Papers on Social Representations, 2, 171-177. Recuperado de http://psych1.lse. ac.uk/psr/PSR1993/2_1993Duvee.pdf

Eibl-Eibesfeldt, I. (1989). Human Ethology. New York: Aldine de Gruyter.

Goldberg, A. (2010). Lesbian and gay parentes and their children: Research on the Family life cycle. Washington, DC: APA.

Goldberg, A., Kashy, D., \& Smith, J. Z. (2012). Gendertyped play behavior in early childhood: Adopted children with lesbian, gay and heterosexual parents. Sex Roles, 67, 503-515. Recuperado de http:// www.ncbi.nlm.nih.gov/pubmed/23420542

Golombok, S. (2013). Families created by reproductive donation: Issues and research. Child Development Perspectives, 7, 61-65. doi: 10.1111/cdep.12015

Golombok, S., Mellish, L., Jennings, S., Casey, P., Tasker, F., \& Lamb, M. (2014). Adoptive gay father families: Parent-child relationships and children's psychological adjustment. Child Development, 85(2), 456-468. doi: 10.1111/cdev.12155

Green, J. L., \& Wallat, C. (1981). Papping instructional conversations. A sociolinguistic ethnography. Em J. L. Green e C. Wallat (Eds.), Ethnography and Language in Educatinal Settings (pp. 161-205). Norwood, NW: ABLEX.

Harris, J. (1999). Diga-me com quem anda... Rio de Janeiro: Objetiva.

Lordelo, E. (1995). Ambiente de desenvolvimento humano: Uma análise a partir do contexto creche (Tese de doutorado em Psicologia não publicada). Instituto de Psicologia, Universidade de São Paulo. São Paulo, SP.

Maccoby, E. (1988). Gender as a social category. Developmental Psychology, 24, 755-765. Recuperado de http://psycnet.apa.org/index.cfm?fa=buy. optionToBuy\&id=1989-08124-001

Morais, M. L. S., \& Otta, E. (2003). Entre a serra e o mar. Em A. M. A. Carvalho, C. M. C. Magalhães,
F. A. R. Pontes \& I. D. Bichara (Eds.), Brincadeira e cultura: viajando pelo Brasil que brinca. (pp. 127-157). São Paulo: Casa do Psicólogo.

Murphy, D. A. (2013). The desire for parenthood: Gay men choosing to become parents through surrogacy. Journal of Family Issues, 34(8), 1104-1124. doi 10.1177/0192513X13484272

Patterson, C. (2009). Children of lesbian and gay parents: Psychology, law, and policy. American Psychologist, 64, 727-736. doi: 10.1037/0003-066X.64.8.727.

Riggs, D. W., \& Due, C. (2010). Gay men, race and surrogacy in Índia: Outskirts: Feminisms Along the Edge, 22. Recuperado de http://www.outskirts.arts.uwa. edu.au/volumes/volume-22/riggs

Sager, Fabio, \& Sperb, Tania M. (1998). O brincar e os brinquedos nos conflitos entre crianças. Psicologia: Reflexão e Crítica, 11(2), 309-326. doi: 10.1590/ S0102-79721998000200010

Scott, J. W. (1989). Gender: A useful category of historical analysis. The American Historical Review, 91(5), 1053-1075. Recuperado de http://www.westga. edu/ history/FacultyUpdated/EMacKinnon/ Spring\%202011\%20Syllabi\%20and\%20Materials/ Gender.pdf

Silva, L. I. C., Pontes, F. A. R., Silva, S. B., Magalhães, C. M. C., \& Bichara, I. D. (2006). Diferenças de gêneros nos grupos de brincadeira na rua: A hipótese de aproximação unilateral. Psicologia, Reflexão \& Crítica, 19(1), 114-121. doi: 10.1590/ S0102-79722006000100016

Stacey, J. (2006). Gay parenthood and the decline of paternity as we knew it. Sexualities, 9(1), 27-55. doi: 10.1177/1363460706060687

Wanderlind, F., Martins, G. D. F., Hansen, J., Macarini, S. M., \& Vieira, M. L. (2006). Diferenças de gênero no brincar de crianças pré-escolares e escolares na brinquedoteca. Paidéia, 16(34), 263-273. doi: 10.1590/S0103-863X2006000200014

Welzer-Lang, D. (2001), A construção do masculino: Dominação das mulheres e homofobia. Revista Estudos Feministas, 9(2), 460-482. Recuperado de http://www.scielo.br/pdf/ref/v9n2/8635.pdf

Recebido em: 16/12/2014

Primeira reformulação em: 15/04/2015 Segunda reformulação em: 05/05/2015

Aprovado em: 19/05/2015 
Nota dos autores:

Apoio: CAPES

Sobre os autores:

Elder Cerqueira-Santos é doutor em Psicologia pela UFRGS, com pós-doutorado em Sexualidade pela University of Toronto, Canadá, professor do PPG em Psicologia Social da UFS, onde coordena o Grupo de Estudos e Pesquisas sobre Sexualidade Humana - SexUs, e pesquisador PQ CNPq.

E-mail: eldercerqueira@gmail.com

Justin Bourne é educador e especialista em Sexualidade Humana, doutor em Psicologia da Personalidade, atua como educador social em projetos de direitos humanos e sexualidade no Canadá e é colaborador da University of Toronto, Canadá.

Contato com os autores:

Elder Cerqueira-Santos

Universidade Federal de Sergipe, Departamento de Psicologia

Av. Marechal Rondon, s/n, Jardim Rosa Elze, São Cristóvão, SE

CEP: 49100-000

Psico-USF, Bragança Paulista, v. 21, n. 1, p. 125-133, jan./abr. 2016 
